\title{
SPURIOUS, ZEROTH-ORDER ENTROPY GENERATION ALONG A KINKED WALL
}

\author{
ERIC VAN DER MAAREL AND BARRY KOREN \\ Center for Mathematics and Computer Science, PO Box 4079, 1009 AB Amsterdam, The Netherlands
}

\section{SUMMARY}

Numerical entropy generation is studied in the case of steady, subsonic Euler flow along a kinked solid wall. For a standard upwind finite volume discretization the numerical entropy error, a component of the global discretization error, appears to be zeroth-order in mesh size. Two possible causes of the zeroth-order entropy error are studied. First an investigation is made of the local truncation error on a kinked grid. Although this error also appears to be zeroth-order in the neighbourhood of the kink, it probably does not cause the zeroth-order entropy error. Next a study is made of the existence of a singularity in the exact solution. Probably, the Euler flow solution is singular at the kink in the wall. The form of this likely singularity is unknown. Therefore the construction of a computational method which uses a priori knowledge about the singularity is not possible. Finally it is shown by numerical experiments that the subsonic Euler flow along a kinked wall still can be computed with vanishing entropy errors by using an appropriate sequence of continuously curved walls which converge to the kinked wall in the limit of zero mesh width.

KEY WORDS Steady Euler equations Subsonic flow Entropy error Local truncation error Global discretization error Singularity

\section{INTRODUCTION}

Numerical approximations of the subsonic Euler flow along a kinked solid wall show an erroneous entropy generation which is virtually independent of the mesh size $h$ of the computational grid. In this paper we study this numerical entropy generation for the steady, twodimensional Euler equations and a perfect gas. The steady, two-dimensional Euler equations can be written as

$$
\frac{\partial \mathbf{f}(\mathbf{q})}{\partial x}+\frac{\partial \mathbf{g}(\mathbf{q})}{\partial y}=\mathbf{0}
$$

with

$$
\mathbf{q}=\left[\begin{array}{l}
\rho \\
\rho u \\
\rho v \\
\rho E
\end{array}\right], \quad \mathbf{f}(\mathbf{q})=\left[\begin{array}{l}
\rho u \\
\rho u^{2}+p \\
\rho u v \\
\rho u H
\end{array}\right], \quad \mathbf{g}(\mathbf{q})=\left[\begin{array}{l}
\rho v \\
\rho u v \\
\rho v^{2}+p \\
\rho v H
\end{array}\right]
$$

In (2) the usual notations have been used: $u$ and $v$ for the velocity components in the $x$ - and $y$-directions respectively, $\rho$ for the density and $p$ for the pressure. $E$ denotes the total energy

0271-2091/91/191113-17\$08.50

(C) 1991 by John Wiley \& Sons, Ltd.

Received May 1990

Revised January 1991 
defined by $E=e+\frac{1}{2}\left(u^{2}+v^{2}\right)$, with $e$ the internal energy, which for a perfect gas can be written as

$$
e=\frac{1}{\gamma-1} \frac{p}{\rho}
$$

with $\gamma$ denoting the ratio of (constant) specific heats.

We proceed by giving a brief outline of the basic discretization method used in this paper: the upwind finite volume method which was first presented in Reference 1 . The method has already been shown to yield good numerical solutions not only for transonic and supersonic flows but also for fully subsonic flows, flows which are often neglected when validating discretization methods for the compressible Euler equations, in particular when these are upwind methods. For extensive evaluations of the present, basic discretization method we refer to References $2-4$, in particular to Reference 2, in which-among others-a standard test case for fully subsonic Euler flow is considered: the NACA 0012 aerofoil at $M_{\infty}=0.63$ and $\alpha=2^{\circ}$.

The steady Euler equations are written in the integral form which is found by integrating (1) over some area $\Omega^{*}$, an arbitrary subdomain of the computational domain $\Omega$ :

$$
\iint_{\Omega^{*}}\left(\frac{\partial \mathbf{f}(\mathbf{q})}{\partial x}+\frac{\partial \mathbf{g}(\mathbf{q})}{\partial y}\right) \mathrm{d} x \mathrm{~d} y=\oint_{\partial \Omega^{*}}\left[\mathbf{f}(\mathbf{q}) n_{x}+\mathbf{g}(\mathbf{q}) n_{y}\right] \mathrm{d} s=\mathbf{0} .
$$

In (4), $\partial \Omega^{*}$ is the boundary of $\Omega^{*}$, and $n_{x}$ and $n_{y}$ are the $x$ - and $y$-components respectively of the outward unit normal at $\partial \Omega^{*}$. The Euler equations are then discretized by requiring (4) to hold for each $\Omega_{i, j}$, i.e. each $(i, j)$ th quadrilateral finite volume in a disjunct division of the computational domain $\Omega, \Omega=\bigcup_{i, j} \Omega_{i, j}$. Given a quadrilateral finite volume division, along $\partial \Omega_{i, j}$ the integral in (4) consists of four parts, each of which with constant $n_{x}$ and $n_{y}$. At each cell face the line integral is approximated by taking the flux functions $\mathbf{f}(\mathbf{q})$ and $\mathbf{g}(\mathbf{q})$ constant and by computing them with Osher's numerical flux function ${ }^{5}$ in the P-variant as proposed by Hemker and Spekreijse. ${ }^{1}$ Denoting the cell face between e.g. $\Omega_{i, j}$ and $\Omega_{i+1, j}$ by $\partial \Omega_{i+1 / 2, j}$ and the numerical flux functions approximating $\mathbf{f}(\mathbf{q})$ and $\mathbf{g}(\mathbf{q})$ by $\mathbf{F}\left(\mathbf{q}^{1}, \mathbf{q}^{\Gamma}\right)$ and $\mathbf{G}\left(\mathbf{q}^{1}, \mathbf{q}^{\mathrm{r}}\right)$, the integral along cell face $\partial \Omega_{i+1 / 2, j}$ can be written as

$$
\begin{aligned}
\int_{\partial \Omega_{i+1 / 2, j}}\left[\mathbf{f}(\mathbf{q}) n_{x}+\mathbf{g}(\mathbf{q}) n_{y}\right] \mathrm{d} s= & {\left[\mathbf{F}\left(\mathbf{q}_{i+1 / 2, j}^{\mathrm{1}}, \mathbf{q}_{i+1 / 2, j}^{\mathrm{r}}\right)\left(n_{x}\right)_{i+1 / 2, j}\right.} \\
& \left.+\mathbf{G}\left(\mathbf{q}_{i+1 / 2, j}^{1}, \mathbf{q}_{i+1 / 2, j}^{\mathrm{F}}\right)\left(n_{y}\right)_{i+1 / 2, j}+\mathbf{O}\left(h^{\mu}\right)\right] l_{i+1 / 2, j},
\end{aligned}
$$

where $l_{i+1 / 2, j}$ is the length of $\partial \Omega_{i+1 / 2, j},\left(n_{x}\right)_{i+1 / 2, j}$ and $\left(n_{y}\right)_{i+1 / 2, j}$ are the components of the outward unit normal at $\partial \Omega_{i+1 / 2, j}, \mathbf{q}_{i+1 / 2, j}^{1}$ and $\mathbf{q}_{i+1 / 2, j}^{\mathrm{f}}$ are the states at the left and right sides of $\partial \Omega_{i+1 / 2, j}$ respectively and $\mu$ is the order of accuracy of the approximation. In this paper we take the state vector $\mathbf{q}_{i, j}$ constant over each cell $\Omega_{i, j}$. Taking for the left and right states at e.g. cell face $\partial \Omega_{i+1 / 2, j}$

$$
\mathbf{q}_{i+1 / 2, j}^{1}=\mathbf{q}_{i, j}, \quad \mathbf{q}_{i+1 / 2, j}^{\mathrm{r}}=\mathbf{q}_{i+1, j},
$$

and similarly for $\mathbf{q}_{i, j+1 / 2}^{1}$ and $\mathbf{q}_{i, j+1 / 2}^{\Gamma}$ the corresponding adjacent volume states, on a smooth grid this yields a first-order-accurate discretization: $\mu=1$.

Boundary conditions are incorporated into the scheme in a way which is consistent with the discretization in the interior of the computational domain. In subsonic flows this requires that three boundary conditions are imposed at inflow and one boundary condition at outflow. (We remark that by just obeying these numbers in subsonic flow computations, mathematical well-posedness is not yet guaranteed. For a study of the mathematical well-posedness of e.g. some 
typical subsonic outlet boundary conditions we refer to Reference 3.) For supersonic flow the number of boundary conditions to be imposed at inflow and outflow is four and zero respectively.

In this paper we will mainly consider flows of which the exact solutions are known to be homentropic. We will make proper use of this knowledge to monitor the quality of numerical solutions obtained for these flows. As is well known, in steady subsonic flow the entropy along a streamline is constant. Hence a first requirement for obtaining a homentropic flow in a numerical computation is to impose a constant entropy at inflow.

We study the entropy error, one of the four components of the global discretization error, for a subsonic Euler flow along a kinked wall (Figure 1). At the inflow boundary the velocity vector and the entropy are imposed. The imposed velocity corresponds to that of an incompressible, irrotational flow along a kinked wall. At outflow the corresponding pressure is imposed. The type of grid used is shown in Figure 2. It consists of $32 \times 32$ finite volumes. Coarser grids $(16 \times 16$ and $8 \times 8)$ are obtained from the finer one by leaving out each second grid line. Entropy errors obtained along the wall are given in Figure 3. The results show that the entropy error is virtually independent of the mesh size; they clearly outline the problem to be studied in this paper. For the sake of clarity we emphasize that the entropy error can be seen as a monitor for the complete global discretization error. Most likely, the other three components of the global discretization error will also be zeroth-order-accurate.

\section{NON-SMOOTHNESS OF THE GRID}

Having noticed the zeroth-order behaviour of the entropy error, it is natural first to investigate the local truncation error of the numerical scheme. In the following we study the effect of the grid's non-smoothness on the local truncation error of the upwind finite volume discretization.

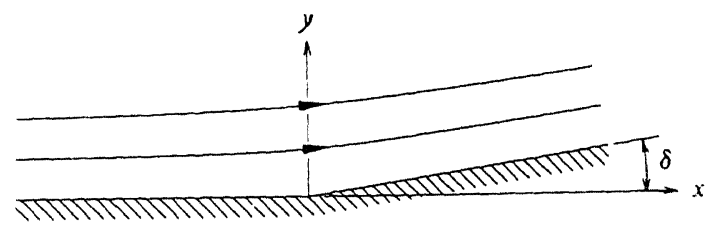

Figure 1. Kinked wall

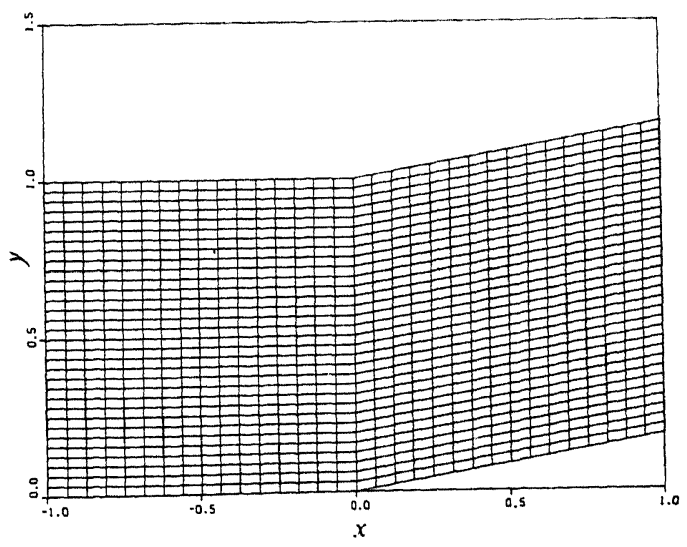

Figure 2. Kinked grid, $32 \times 32$ 


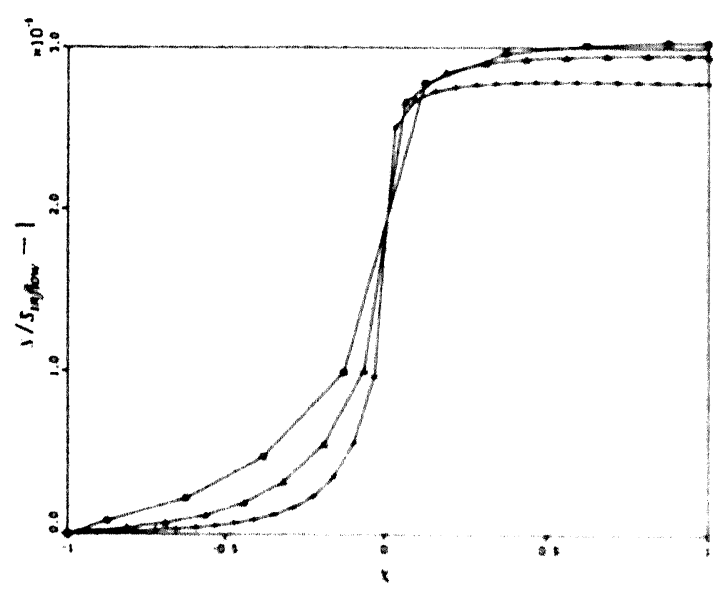

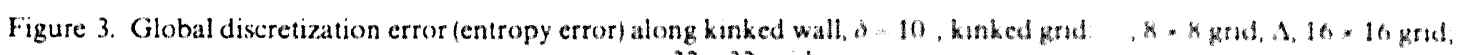
$+.32 \times 32 \mathrm{grid}$

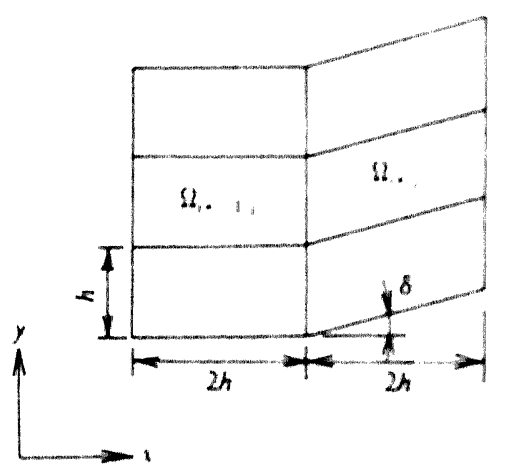

Figure 4 Cieometry at bink

\subsection{Local truncation error}

In Section 1 we mentioned that the flux functions at e.g. cell face $i \Omega_{j}, 2$, are approxumated by

$$
(\mathbf{f}(\mathbf{q}))_{i+1 / 2 . j}=\mathbf{F}\left(\mathbf{q}_{i, j}, \mathbf{q}_{i+1, j}\right)+\mathbf{O}(h), \quad(\mathbf{g}(\mathbf{q}))_{i+1, j}=\mathbf{G}\left(\mathbf{q}_{i, j}, \mathbf{q}_{i, 1, j}\right) \mathbf{O}(\mathbf{h})
$$

Let $\mathbf{q}$ denote the exact, continuous solution of the Fuler equations in the doman susced by the grid as shown in Figure 2 and let $\mathbf{q}_{i . j}$ denote the discrete value of $\mathbf{q}$ in the centre of $\Omega_{,}$, Then for $\Omega_{1 *}, 1$, a cell just behind the kink in the grid (Figure 4$)$, the system of discretued equatuns reats

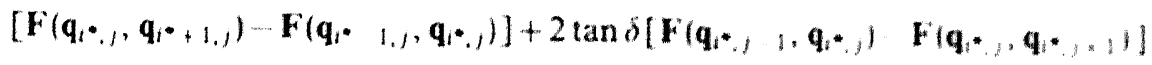

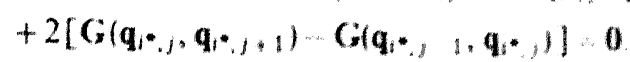

Taylor series expansion around the centre of $\Omega_{1 *}$, yields for the first term in $(8)$

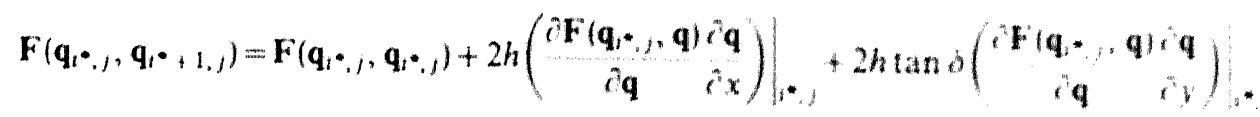


Similar formulae for the other terms in (8) and

$$
\left.\frac{\partial \mathbf{F}}{\partial x}\right|_{i, j}=\left.\left(\frac{\partial \mathbf{F}\left(\mathbf{q}, \mathbf{q}_{i, j}\right)}{\partial \mathbf{q}} \frac{\partial \mathbf{q}}{\partial x}\right)\right|_{i, j}+\left.\left(\frac{\partial \mathbf{F}\left(\mathbf{q}_{i, j}, \mathbf{q}\right)}{\partial \mathbf{q}} \frac{\partial \mathbf{q}}{\partial x}\right)\right|_{i, j}
$$

yield then as modified equation for (8)

$$
\left.\frac{\partial \mathbf{F}}{\partial x}\right|_{i^{*}, j}+\left.\frac{\partial \mathbf{G}}{\partial y}\right|_{i^{*}, j}-\left.\frac{1}{2} \tan \delta\left(\frac{\partial \mathbf{F}\left(\mathbf{q}, \mathbf{q}_{i^{*}, j}\right)}{\partial \mathbf{q}} \frac{\partial \mathbf{q}}{\partial y}\right)\right|_{i^{*}, j}=\mathbf{O}(h)
$$

From (11) we see that the discretization has a zeroth-order local truncation error. The error vanishes for $\delta=0$ (of course) or for fully one-sided upwind discretization from the right. (In the latter case $\mathbf{F}\left(\mathbf{q}, \mathbf{q}_{i^{*}, j}\right)=\mathbf{f}\left(\mathbf{q}_{i^{*}, j}\right)$ and hence $\partial \mathbf{F}\left(\mathbf{q}, \mathbf{q}_{i^{*}, j}\right) / \partial \mathbf{q}=\mathbf{0}$.) A similar result is obtained when we analyse the local truncation error for a cell $\Omega_{i^{*}-1, j}$ just in front of the kink:

$$
\left.\frac{\partial \mathbf{F}}{\partial x}\right|_{i^{*}-1, j}+\left.\frac{\partial \mathbf{G}}{\partial y}\right|_{i^{*}-1, j}+\left.\frac{1}{2} \tan \delta\left(\frac{\partial \mathbf{F}\left(\mathbf{q}_{i^{*}-1, j}, \mathbf{q}\right)}{\partial \mathbf{q}} \frac{\partial \mathbf{q}}{\partial y}\right)\right|_{i^{*}-1, j}=\mathbf{O}(h) \text {. }
$$

Thus for a cell just in front of the kink the zeroth-order local truncation error vanishes for a fully left-sided upwind discretization or, trivially, for vanishing $\delta$.

To verify the analysis, we substitute an exact solution of the Euler equations into the discretized equations. For the exact solution we take a supersonic, non-uniform, parallel flow

$$
(u, v, \rho, p)^{\mathrm{T}}=\left(u_{0}+u_{1} y, 0, \rho_{0}, p_{0}\right)^{\mathrm{T}},
$$

with $u_{0}, u_{1}, \rho_{0}, p_{0}$ constant and $u_{0}, u_{1}$ both positive. No solid wall boundary is involved in this flow. Since the flow solution has a gradient in the $y$-direction, for $\delta \neq 0$ we expect to have a zeroth-order local truncation error. Since the flow is supersonic from the left, we should find zeroth-order local truncation errors only in cells just behind the kink. Numerical results show that zeroth-order local truncation errors are indeed concentrated around the cell centres just behind the kink (Figure 5). If we take the same supersonic flow in the opposite direction $\left(u_{0}<0, u_{1}<0\right)$, as expected, zeroth-order local truncation errors are concentrated around cell centres just in front of the kink (Figure 6(a)). In subsonic flow (Figure 6(b)) we find zeroth-order local truncation errors both in front of and behind the kink, as could be expected from (11) and

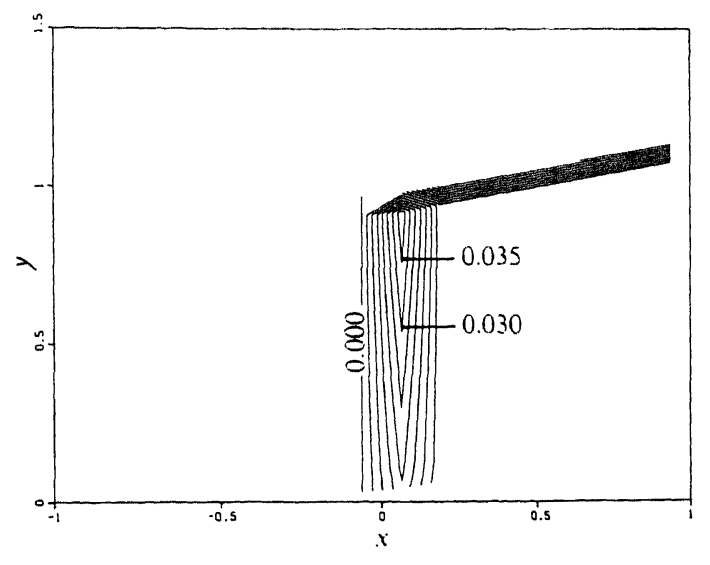

a. On $16 \times 16$-grid.

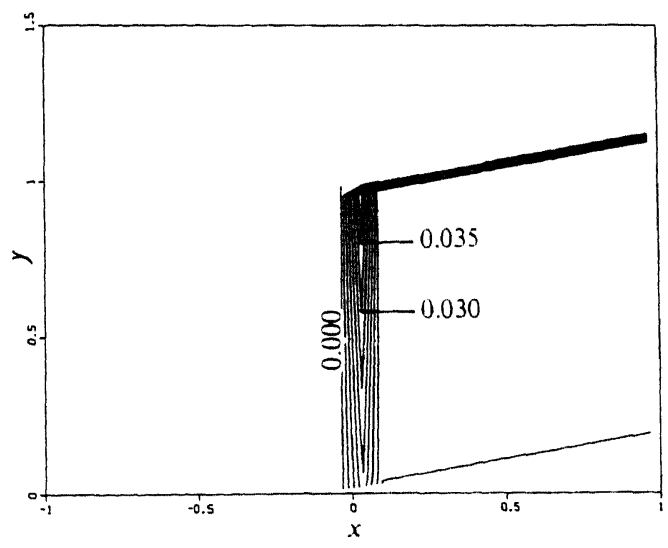

b. On $32 \times 32$-grid.

Figure 5. Local truncation error (residual energy equation), supersonic flow from the left, $\delta=10^{\circ}$ (without wall) 


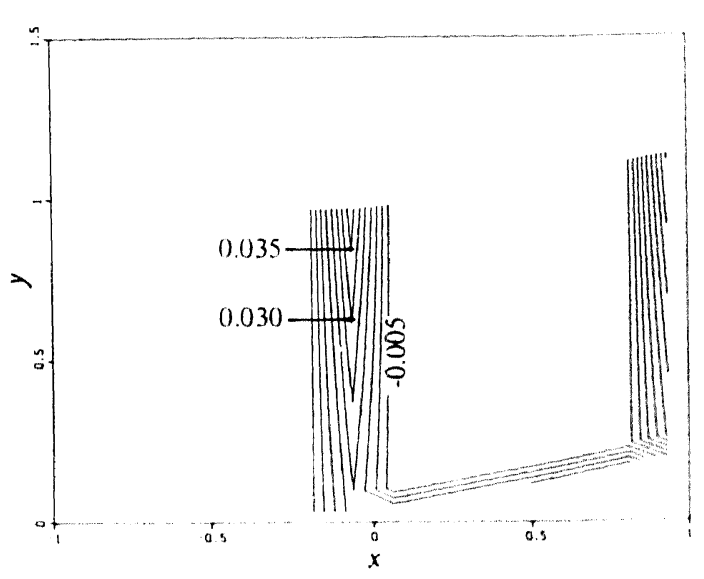

a. Supersonic flow from the right.

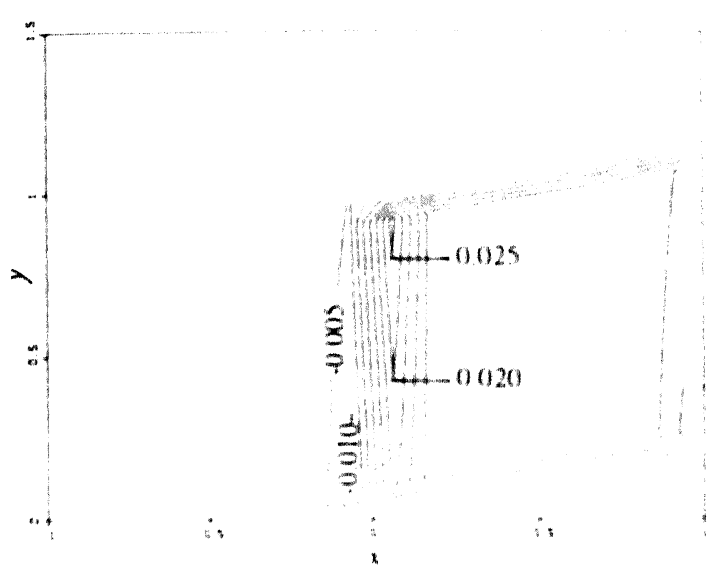

h. Subsonic flow from the left

Figure 6. Local truncation error (residual energy equation, $16 \times 16$ gnd, $\delta \ldots 10$ (without wall)

(12), since in subsonic flow the numerical flux function depends on both the left and right states (As can be seen from both Figures 5 and 6, along a part of the boundary zeroth-order errors are found as well. Apparently, the boundary of the computational domain causes local truncation errors as though the grid were kinked at the boundary. It can be shown analytically that at the boundary the behaviour of the local truncation error with respect to the upwind direction is indeed identical to that at the kink in the grid.)

Remark. The local truncation errors presented in Figures 5 and 6 are those of the Fuler equations discretized by a finite volume method and hence they are derived from integrated quantities. In order to compare integrated residual fields for a family of grids, they have to be scaled with their corresponding volume areas; therefore in both figures we have given the isolevels of $\iint_{\Omega_{1, j}}$ (residual) $\mathrm{d} x \mathrm{~d} y / \iint_{\Omega_{1, j}} \mathrm{~d} x \mathrm{~d} y$.

\subsection{New discretization}

The zeroth-order local truncation errors as derived in Section 2.1 only involve derivatives in the $y$-direction, i.e. the direction in which the cells behind the kink are shifted. In this subsection we try to remove these errors by enlarging the stencil of the discretizatuon. Since for this purpose we only need to introduce extra derivatives in the $y$-direction, we simply widen the stench in the $y$-direction. A new flux across $\partial \Omega_{\mathrm{i}^{*}+1 / 2, j}$ is introduced by taking a weighted mean of the existing

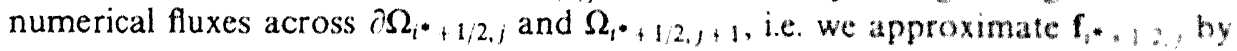

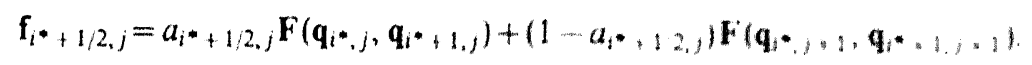

The same is done for the approximation of the flux across $i \Omega_{i}$.

$$
\mathbf{f}_{i *-1 / 2, j}=a_{i *-1 / 2, j} \mathbf{F}\left(\mathbf{q}_{i * 1, j}, \mathbf{q}_{i *, j}\right)+\left(1-a_{i} * 12, j\right) \mathbf{F}\left(\mathbf{q}_{i} * 1,3,1, \mathbf{q}_{i}^{*}, \ldots, 1\right.
$$

Substitution of (14) and (15) into the discrete version of (4) and application of Taylor senes expansion around the centre of $\Omega_{i^{*}, j}$ then yields as modified equation

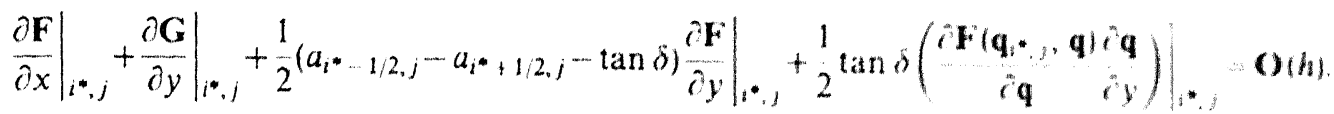


In a fully one-sided upwind discretization from the left (i.e. in a supersonic flow from the left) the fourth term in (16) is equal to zero by itself. The third term can be made equal to zero by choosing the weights such that they satisfy $a_{i^{*}-1 / 2, j}-a_{i^{*}+1 / 2, j}-\tan \delta=0$. Thus, applying this new discretization to (13), in the case of supersonic flow from the left the fourth term in (16) drops automatically. Using the old discretization for the flow, there was no zeroth-order local truncation error in $\Omega_{i^{*}-1, j}$ (see Figure 5). To ensure that there is still no zeroth-order local truncation error in $\Omega_{i^{*}-1, j}$, in that volume we still use the old discretization (i.e. $a_{i^{*}-1 / 2, j}=1$ ). Hence, by choosing

$$
a_{i^{*}-1 / 2, j}=1, \quad a_{i^{*}+1 / 2, j}=1-\tan \delta,
$$

the zeroth-order local truncation error in $\Omega_{i^{*}, j}$ should vanish for supersonic flow from the left without introducing a zeroth-order error in $\Omega_{i^{*}-1, j}$. However, this new discretization for $i=i^{*}$ does introduce zeroth-order local truncation errors in the cells downstream of $\Omega_{i^{*}, j}$. In order to remove these errors, we apply the new discretization in all cells downstream of the kink. (Notice that for $\delta=0$ the new discretization equals the old discretization.) In Figure 7 now, local truncation error distributions are given as found by substitution of the exact solution (13) into the new system of discretized equations. We observe that the local truncation error indeed behaves first-order.

In subsonic flow the Riemann solver is not fully one-sided and as a consequence the zerothorder local truncation error in (16) can be removed only by making the sum of the third and fourth terms in (16) vanish. In that case the weights $a_{i^{*}-1 / 2, j}$ and $a_{i^{*}+1 / 2, j}$ become dependent on the solution. Thus in subsonic flow the zeroth-order local truncation error cannot be removed without introducing a significantly more complicated discretization. Before making any attempt to construct a discretization of this kind, we will first investigate to what extent the zeroth-order local truncation error actually causes the zeroth-order global discretization error.

\subsection{Entropy error on a kinked grid not involving a wall}

In this subsection we study the entropy error on a kinked grid without a wall. The purpose is to find out whether or not the zeroth-order entropy error, as found in the approximation of the flow

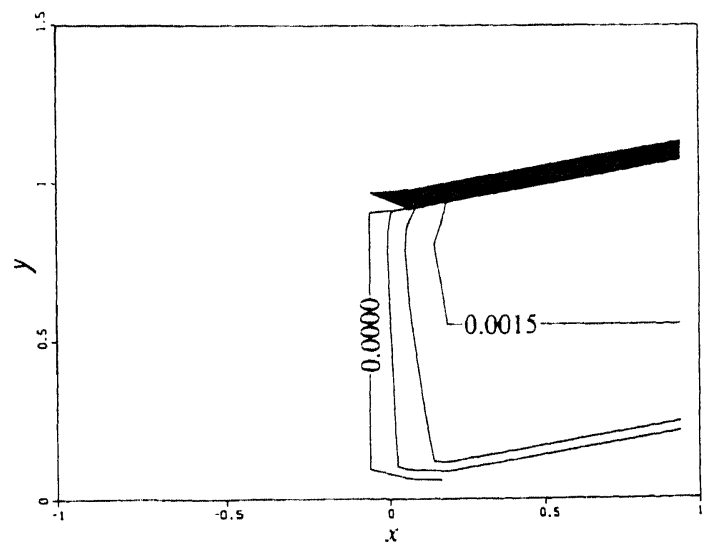

a. On $16 \times 16$-grid.

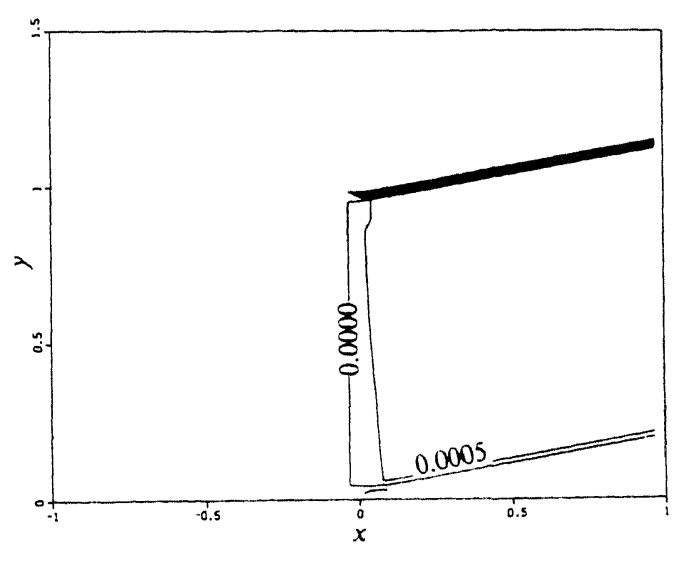

b. On $32 \times 32$-grid.

Figure 7. Local truncation error (residual energy equation), supersonic flow from the left, $\delta=10^{\circ}$ (without wall), new discretization 


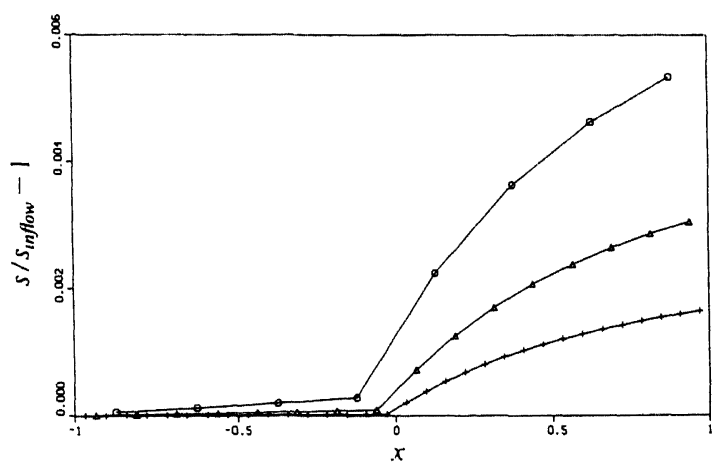

Figure 8. Global discretization error (entropy error) in bottom row of cells, subsonic flow from the left, $\delta=10^{\circ}$ (without wall), old discretization: $0,8 \times 8$ grid; $\Delta, 16 \times 16$ grid;,$+ 32 \times 32$ grid

along a kinked wall (Figure 3), is caused by the zeroth-order local truncation error on a kinked grid. We consider the old discretization and a subsonic version of (13) as the exact flow field. However, here we will not use this exact flow field to evaluate the local truncation error but instead to solve the discretized equations by imposing the exact flow field through the boundary conditions. If for the flow without a wall on the kinked grid shown in Figure 2 zeroth-order solution errors-i.e. global discretization errors-are found, then these may be caused by the zeroth-order local truncation error. However, if we do not find zeroth-order global discretization errors in the approximate solution, assuming that the parallel flow considered is not too trivial, the kinked grid and the resulting zeroth-order local truncation errors do not cause zeroth-order global discretization errors.

Results are given in Figure 8, which shows the entropy error in the bottom row of cells for different mesh sizes. The results clearly show that the entropy error becomes approximately twice as small when the mesh size is made twice as small. This first-order entropy behaviour seems to imply that the zeroth-order local truncation error which occurs in the case of a kinked grid is indeed harmless. Therefore we postulate that the zeroth-order entropy error as found in the approximation of the subsonic flow along a kinked wall is not caused by the zeroth-order local truncation error.

\section{ENTROPY ERRORS IN A SLIGHTLY DIFFERENT SITUATION}

\subsection{Entropy error along a smooth wall}

In this subsection we study the entropy error in the flow along a continuously curved wall. This is done in order to make sure that the solid wall boundary condition, or rather its numerical modelling, does not cause the zeroth-order entropy error. The shape of the wall which we will use is given by

$$
y_{\mathrm{w}}(x)= \begin{cases}0, & x \leqslant-\frac{1}{2} l, \\ \tan \delta \frac{\left(x+\frac{1}{2} l\right)^{3}}{l^{2}}-\frac{1}{2} \tan \delta \frac{\left(x+\frac{1}{2} l\right)^{4}}{l^{3}}, & -\frac{1}{2} l<x \leqslant \frac{1}{2} l, \\ (\tan \delta) x, & x>\frac{1}{2} l .\end{cases}
$$


Here $l$ is the length of the curved part of the wall (Figure 9) and $\delta$ is the angle between the positive $x$-direction and the uncurved part of the wall at $x \geqslant \frac{1}{2} l$ (Figure 9). The wall is defined in such a way that $y_{\mathrm{w}}(x)$ is a $C^{2}$-function. The type of grid used is shown in Figure 10. In Figure 11, for $l=1$ and three mesh sizes, the entropy error along the wall is given. We see that the entropy error is first-order in mesh size. Since the scheme is the first-order-accurate on smooth grids, this is what we were expecting. The results make it perfectly clear that no zeroth-order entropy errors are introduced by the solid wall boundary condition treatment.

\subsection{Entropy error for other types of grids}

In this subsection we study the entropy error for two cases in which the Euler flow along the kinked wall is approximated on types of grids which are essentially different from the simple kinked grid (Figure 2) as applied in the previous sections. First a grid is used which is only kinked at the wall (Figure 12(a)). In the interior of the domain it is smooth and orthogonal; its grid lines are hyperbolae. We will call this grid the hyperbolic grid. Since this grid is smooth, except at a single point, it will not cause zeroth-order local truncation errors along the complete $i$ th grid line as does the simple kinked grid. The other grid, shown in Figure 12(b), does not contain any kink at all, but it has degenerated quadrilaterals located at the kink. We call this grid the circular grid. Entropy errors along the wall as obtained on these grids are shown in Figure 13. For both grids we immediately see that the entropy error is also zeroth-order at the kink. A peculiarity of the results obtained on the circular grid, in comparison to those obtained on the hyperbolic and kinked grids, is that the entropy error along the wall decreases behind the kink. We think that this

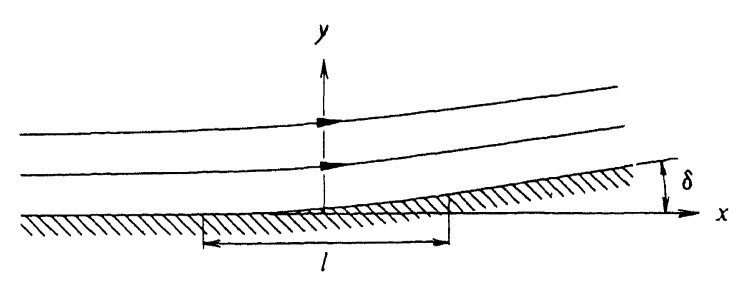

Figure 9. Continuously curved wall

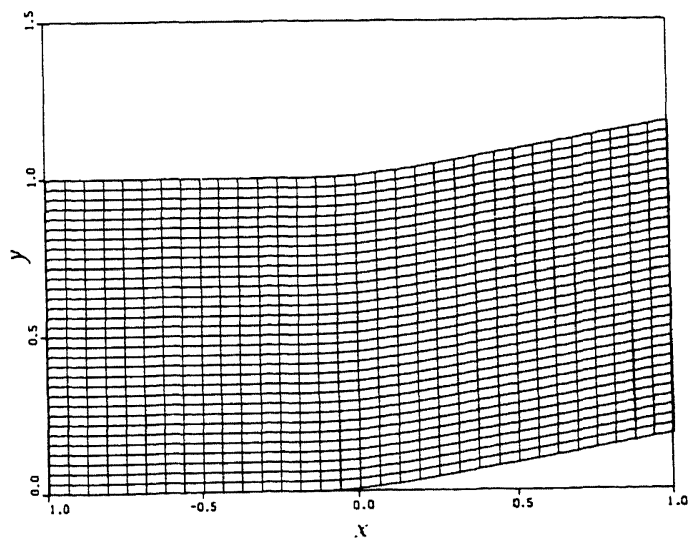

Figure 10. Continuously curved grid, $32 \times 32$ 


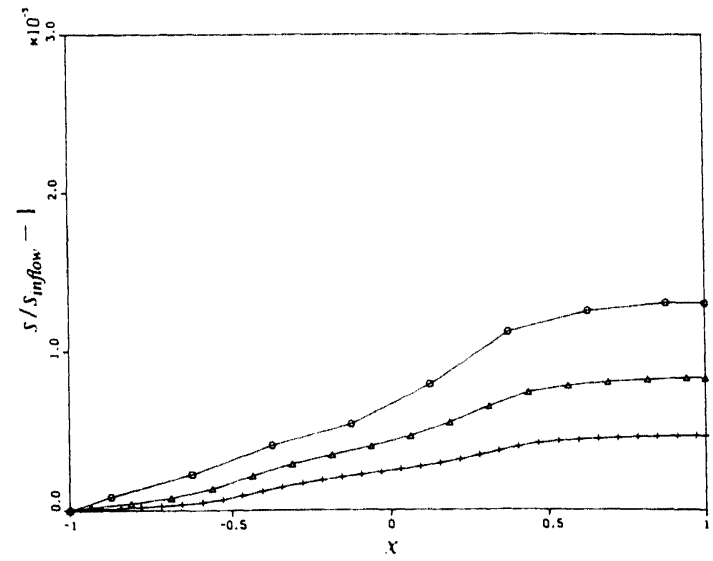

Figure 11. Global discretization error (entropy error) along continuously curved wall, $\delta=10^{\circ}: \bigcirc, 8 \times 8$ grid; $\Delta, 16 \times 16$ grid;,$+ 32 \times 32$ grid

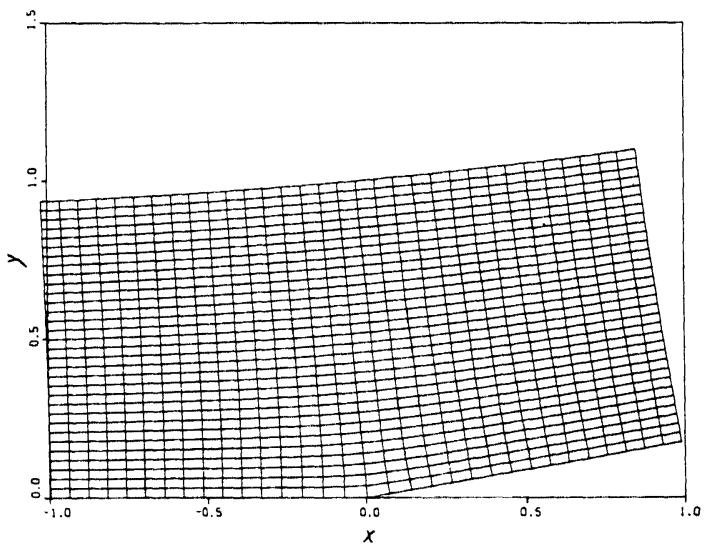

a. Hyperbolic grid.

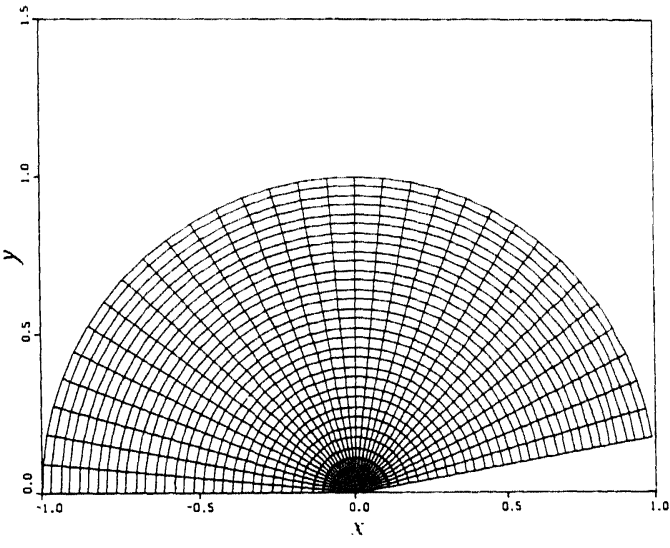

b. Circular grid.

Figure 12. Other types of grids, $32 \times 32$

phenomenon, which we already know from aerofoil flow computations (see e.g. Reference 2), is caused by the increasing mesh width and hence by the increasing amount of crosswise numerical diffusion, when going in the streamwise direction behind the kink, on this circular grid.

\section{SINGULARITY IN THE SOLUTION}

In Section 2.3 we found that the zeroth-order entropy error is probably not caused by the non-smoothness of the grid, in Section 3.1 we found that the error is not caused by the implementation of the solid wall boundary condition and in Section 3.2 we found that it is also not caused by the use of a specific type of grid. In this section we will consider another possible (and now most likely) cause of the error: a singularity (i.e. a non-differentiability) in the (exact, continuous) solution at the kink. 


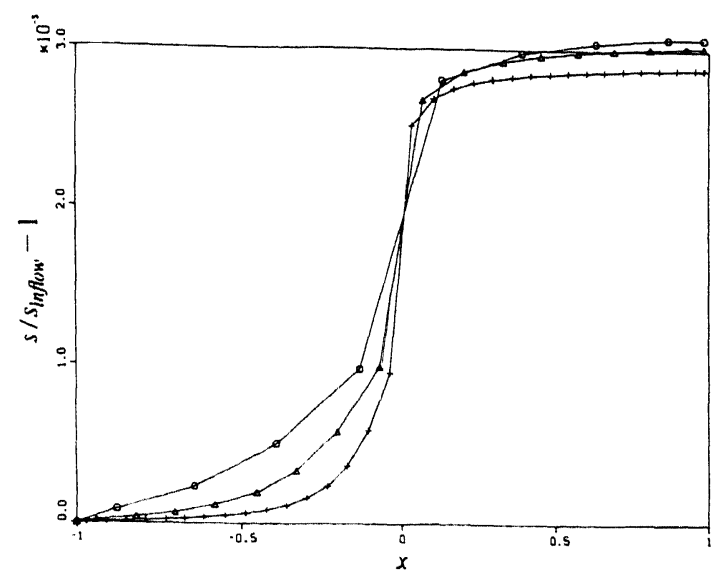

a. On hyperbolic grids.

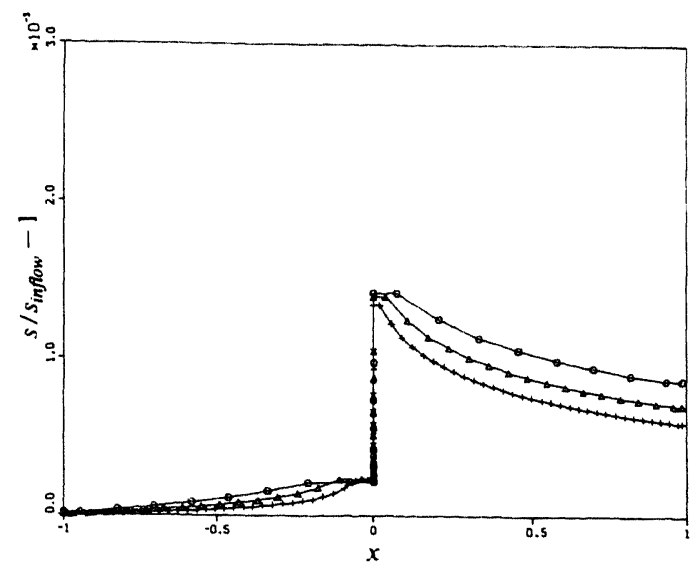

b. On circular grids.

Figure 13. Global discretization error (entropy error) along kinked wall, $\delta=10^{\circ}: 0,8 \times 8$ grid; $\Delta, 16 \times 16$ grid;,$+ 32 \times 32$ grid

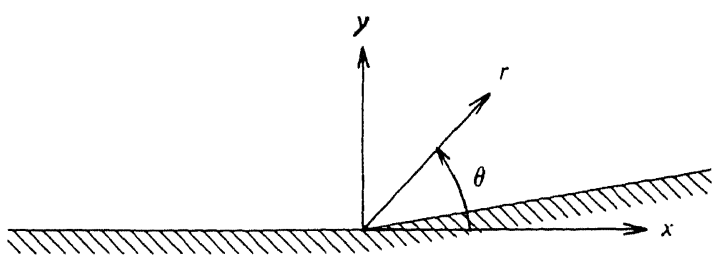

Figure 14. Polar co-ordinates at kink

\subsection{Incompressible potential flow}

For the incompressible potential flow along a kinked wall the exact solution of the continuous potential flow equation is known to be singular. In polar $(r, \theta)$ co-ordinates (Figure 14) for $\delta \leqslant \theta \leqslant \pi$ the velocity field is described by

$$
u=U r^{\delta /(\pi-\delta)} \cos \left(\theta-\frac{\theta-\delta}{\pi-\delta} \pi\right), \quad v=U r^{\delta /(\pi-\delta)} \sin \left(\theta-\frac{\theta-\delta}{\pi-\delta} \pi\right),
$$

where $U$ is a constant. For $0<\delta<\pi / 2$ the velocity field clearly has a singularity at $r=0$. (There, it is not differentiable.)

\subsection{Incompressible potential flow with compressible Euler equations and source term}

In this subsection we investigate whether or not a singularity of the form $r^{\alpha}, 0<\alpha<1$, gives rise to errors which are similar to those found in the previous entropy distributions. We do this by approximating the incompressible potential flow field by numerically solving the compressible Euler equations with a proper source term. (Since the exact solution is known, we can easily evaluate the corresponding global discretization error.) The source term for the equations is found by substituting the exact, incompressible potential flow solution into the continuous 
compressible Euler equations. The incompressible potential flow solution satisfies the "compressible Euler continuity' and 'compressible Euler momentum' equations, but the 'compressible Euler energy' equation is not satisfied. Therefore we (only) need a source term for the energy equation:

$$
\frac{\partial(\rho u H)}{\partial x}+\frac{\partial(\rho v H)}{\partial y}=-\frac{1}{\gamma-1} \rho U^{3} \frac{\delta}{\pi-\delta} r^{-(\pi-4 \delta) /(\pi-\delta)} \cos \left(\frac{\theta-\delta}{\pi-\delta} \pi\right), \quad \delta \leqslant \theta \leqslant \pi .
$$

In order not to let zeroth-order errors be introduced by the source term, we take $\delta=\pi / 4$. For the computation we apply the existing, multigrid-based solution method ${ }^{1}$ to the first-order discretized Euler equations supplied with a second-order approximation of the source term (20).

In Figure 15 numerical results are presented as obtained on the hyperbolic grid. The results shown are the errors in the entropy-like quantity $z=\ln \left(p / \rho^{\gamma}\right)$. (Notice that the function $p / \rho^{\gamma}$ in the exact solution is not a constant.) The figure shows that at some distance from the kink the error in the numerical approximation to this solution (i.e. the global discretization error) is first-orderaccurate, but it becomes zeroth-order-accurate in the vicinity of the kink. From this we conclude that when the solution of the exact equations has a singularity at $r=0$, the global discretization error is zeroth-order at $r=0$. In our numerical approximation of the subsonic, compressible Euler flow along a kinked wall we have a zeroth-order global discretization error at the kink. Thus it is likely that the exact solution of the subsonic compressible Euler equations for the flow along a kinked wall also has a singularity at the kink.

\subsection{Transformation of variables and equations}

In this subsection we investigate whether for compressible Euler flow we can transform the equations in such a way that only smooth (i.e. non-singular) functions remain to be approximated. Herewith we strive for a minor modification of the existing numerical method. We assume that the velocity field of the subsonic, compressible Euler flow along a kinked wall has a singularity similar to that of the incompressible potential flow. In the previous subsection it was suggested that if this is the correct form of the singularity, the approximate solution obtained by solving the discretized Euler equations should have a zeroth-order global discretization error. By extracting such a singularity, one may hope to easily remove this error.

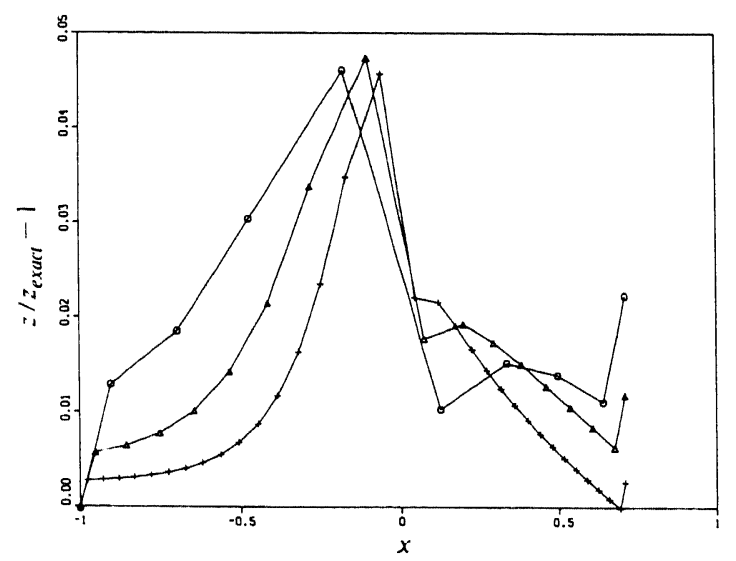

Figure 15. Global discretization error ('entropy' error $z / z_{\text {exact }}-1$ ) along wall, incompressible potential flow, hyperbolic grid, $\delta=\pi / 4: 0,8 \times 8$ grid; $\Delta, 16 \times 16$ grid;,$+ 32 \times 32$ grid 
Assume that $u$ and $v$ can be written as

$$
u=U r^{\alpha}, \quad v=V r^{\alpha},
$$

where $U$ and $V$ are smooth functions of $x$ and $y$ and where, following (19), $\alpha=\delta /(\pi-\delta)$, $0<\delta<\pi / 2$. (Thus $u$ and $v$ are singular at $r=0$.) Further, assume that the exact Euler flow is homentropic and isenthalpic. Then along the wall we have for the speed of sound

$$
c^{2}=c_{0}^{2}-\frac{\gamma-1}{2}\left(U^{2}+V^{2}\right) r^{2 \alpha}=C_{0}^{2}-C_{1}^{2} r^{2 \alpha},
$$

with $C_{0}$ a constant and $C_{1}$ a smooth function. If we assume that the enthalpy changes smoothly from one streamline to another, then (22) is valid everywhere in the flow field, with both $C_{0}$ and $C_{1}$ smooth functions of $x$ and $y$. Furthermore, $C_{1}$ is a known function of $U$ and $V$. With $s=p / \rho^{\gamma}$ constant we find for the density

and for the pressure

$$
\rho=(\gamma s)^{-1 /(\gamma-1)}\left(C_{0}^{2}-C_{1}^{2} r^{2 \alpha}\right)^{1 /(\gamma-1)}=\left(R_{0}-R_{1} r^{2 \alpha}\right)^{1 /(\gamma-1)}
$$

$$
p=\left(\gamma^{\gamma} s\right)^{-1 /(\gamma-1)}\left(C_{0}^{2}-C_{1}^{2} r^{2 \alpha}\right)^{\gamma /(\gamma-1)}=\left(P_{0}-P_{1} r^{2 \alpha}\right)^{\gamma /(\gamma-1)} .
$$

Here $R_{0}, R_{1}, P_{0}$ and $P_{1}$ are smooth functions of $x$ and $y$, and $R_{1}$ and $P_{1}$ are known functions of $U$ and $V$. Next we can transform the Euler equations (1) and (2) into equations to be satisfied by our new, smooth variables $U, V, R_{0}$ and $P_{0}$. Substitution of (21)-(24) into e.g. $\mathbf{f}(\mathbf{q})$ as given in (2) yields

$$
\mathbf{f}(\mathbf{q})=\left[\begin{array}{l}
\rho u \\
\rho u^{2}+p \\
\rho u v \\
\rho u H
\end{array}\right]=\left[\begin{array}{l}
U\left(R_{0} r^{\alpha(\gamma-1)}-R_{1} r^{\alpha(\gamma+1)}\right)^{1 /(\gamma-1)} \\
U^{2}\left(R_{0} r^{2 \alpha(\gamma-1)}-R_{1} r^{2 \alpha \gamma}\right)^{1 /(\gamma-1)}+\left(P_{0}-P_{1} r^{2 \alpha}\right)^{\gamma /(\gamma-1)} \\
U V\left(R_{0} r^{2 \alpha(\gamma-1)}-R_{1} r^{2 \alpha \gamma}\right)^{1 /(\gamma-1)} \\
U s \frac{\gamma}{\gamma-1}\left(R_{0}^{2} r^{\alpha(\gamma-1)}-R_{0} R_{1} r^{\alpha(\gamma+1)}\right)^{1 /(\gamma-1)}
\end{array}\right]
$$

Unfortunately, the transformed fluxes are complicated non-linear functions of the dependent variables $U, V, R_{0}$ and $P_{0}$. The corresponding system of discretized equations cannot be solved by the existing method for the Euler equations, nor by a slightly modified version of it. Apart from the fact that the transformed equations require an ambitious modification of the existing numerical method, it is not yet certain whether or not the assumptions made on the singularity are correct. Therefore we refrain from a further investigation of the transformed equations.

\section{DISCRETIZATION OF THE KINKED WALL BY A SEQUENCE OF SMOOTH WALLS}

In Section 3.1 we computed the Euler flow along a continuously curved wall. There the length $l$ of the continuously curved wall segment (Figure 9) was the same for all three grids considered. In this section we recompute the flow along a continuously curved wall as given by (18), but now we decrease $l$ together with the mesh size: $l=O\left(h^{\lambda}\right), \lambda>0$. For finite $\lambda$ it is clear that for $h \rightarrow 0$ the wall becomes kinked. The results in Sections 1 and 3.1 can be considered as those for the limit cases $\lambda=\infty$ and $\lambda=0$ respectively. The number of cells, $N$, lying along the curved part of the wall is $N=l / h$. Hence with $l=O\left(h^{\lambda}\right)$ we have $N=O\left(h^{\lambda-1}\right)$. Thus for $\lambda<1, N$ increases with decreasing 
$h$ and for $\lambda>1$ it decreases. In the latter case for $h \rightarrow 0$ we find that at the kink there is only one grid line. In that case we would arrive at a similar situation as for $\lambda=\infty$, the situation with zeroth-order entropy error. Thus, looking for decreasing entropy errors for decreasing mesh size, we must take $\lambda<1$.

In Figure 16 we show the behaviour of the entropy error when $l$ decreases as a function of $h$. Here for $l$ we take $l=c_{1} h^{\lambda}$, with $c_{1}$ constant, and for the maximum norm of the entropy error we assume the form $\left\|s / s_{\text {inflow }}-1\right\|_{L_{\infty}}=c_{2} h^{\mu}, c_{2}$ constant. Based on numerical experiments with $c_{1}=1$ on $16 \times 16,32 \times 32$ and $64 \times 64$ grids, $\mu$ has been determined for different values of $\lambda$. As already known, for $\lambda=0$ we find $\mu \rightarrow 1$ for $h \rightarrow 0$. The message of Figure 16 is that the flow along a continuously curved wall can indeed be used to approximate the flow along a kinked wall. For any $\lambda \in] 0,1$ [ in the limit $h \rightarrow 0$ the curved wall becomes kinked and the entropy error vanishes, because for any $\lambda$ in that range it appears that $\left\|s / s_{\text {inflow }}-1\right\|_{L_{\infty}}=O\left(h^{\mu}\right), \mu>0$.

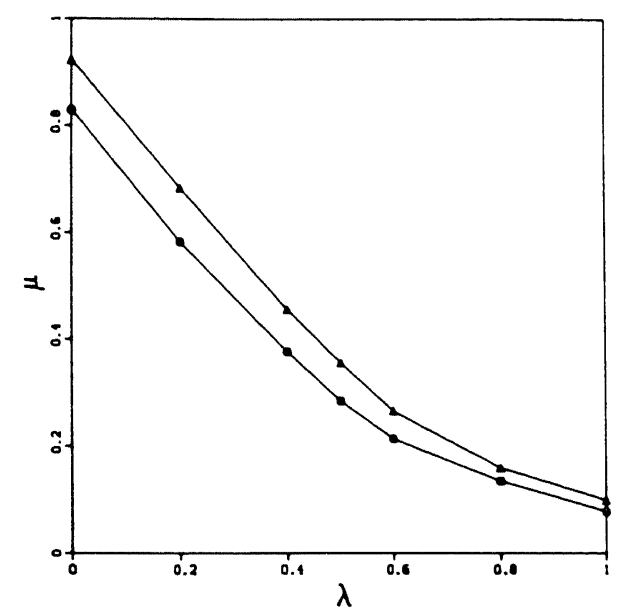

Figure 16. Order behaviour of global discretization error (entropy error), $\delta=10^{\circ}$, shrinking rounded kink: $\bigcirc, 16 \times 16$ grid $/ 32 \times 32$ grid; $\Delta, 32 \times 32$ grid $/ 64 \times 64$ grid

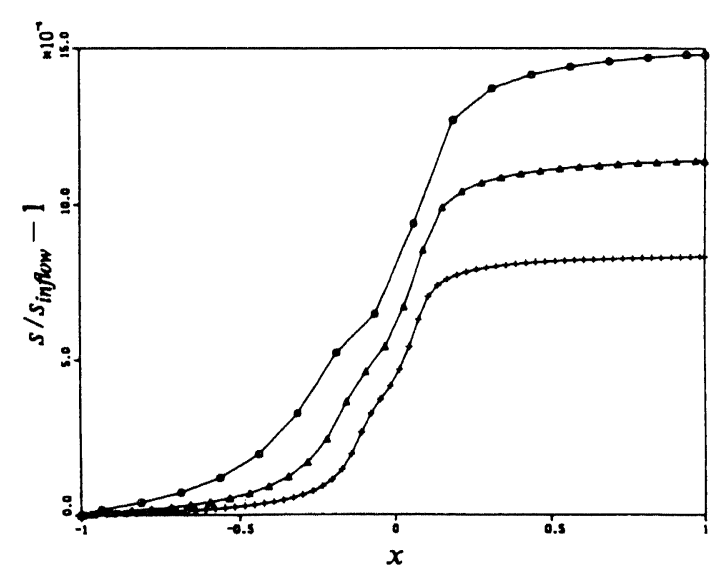

Figure 17. Global discretization error (entropy error) along wall with shrinking rounded kink, $\lambda=0 \cdot 4, \delta=10^{\circ}: 0,16 \times 16$ grid; $\Delta, 32 \times 32$ grid;,$+ 64 \times 64$ grid 
If we want to let the entropy error disappear at the same rate as $l$, then from Figure 16 we find that we should take $\lambda \approx 0 \cdot 4$. In Figure 17 we give the entropy error distributions along the wall as obtained for $\lambda=0.4$ on $16 \times 16,32 \times 32$ and $64 \times 64$ grids. Given the rather low order of accuracy, $\mu(\lambda=0 \cdot 4) \approx 0 \cdot 4$, reduction of the entropy error below some required tolerance level may become expensive when applying global grid refinements solely. The remedy in avoiding too high computational costs lies in applying local grid refinements. As an example, in Figure 18 we give results obtained on a $32 \times 32$ grid enriched with local refinements, still with $\lambda=0.4$. The refinement criterion applied here is based on the gradient of the entropy error in the streamline direction: $\mathbf{w} \cdot \nabla s /\|\mathbf{w}\|_{L_{2}}, \mathbf{w}=(u, v)^{\mathrm{T}}$. For a detailed description of the underlying solution-adaptive multigrid method we refer to Reference 6 . In Figure 19 we give the locally adapted grid corresponding to the most accurate result from Figure 18: the $32 \times 32$ grid with four additional levels of local refinement on top. Notice that for decreasing mesh width the refined regions get smaller and also closer to the corner, which indicates that local refinement (combined with the present smooth discretizations of the kinked wall) is indeed a good possibility for reducing the entropy error without excessively increasing the computational costs. Finally, in Table I for the three grids considered in Figure 18 we give an impression of how the rounded corner converges to the kink for decreasing mesh width.

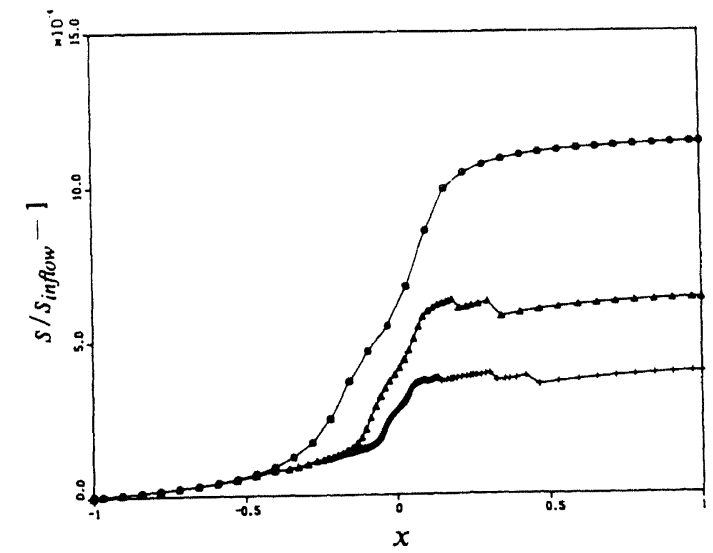

Figure 18. Global discretization error (entropy error) along wall with shrinking rounded kink, $\lambda=0.4, \delta=10^{\circ}: 0,32 \times 32$ Frid without local refinement; $\Delta, 32 \times 32$ grid with two levels of local refinement;,$+ 32 \times 32$ grid with four levels of local grid without local refinement; $\Delta, 32 \times 32$ grid with two levels
refinement

Table I. Geometrical data, rounded corners, $\lambda=0 \cdot 4$

\begin{tabular}{lcr}
\hline Grid & $l$ & $N$ \\
\hline $32 \times 32$ grid without local refinement & 0.3299 & 5 \\
$32 \times 32$ grid with two levels of local refinement & 0.1895 & 12 \\
$32 \times 32$ grid with four levels of local refinement & 0.1088 & 28 \\
\hline
\end{tabular}




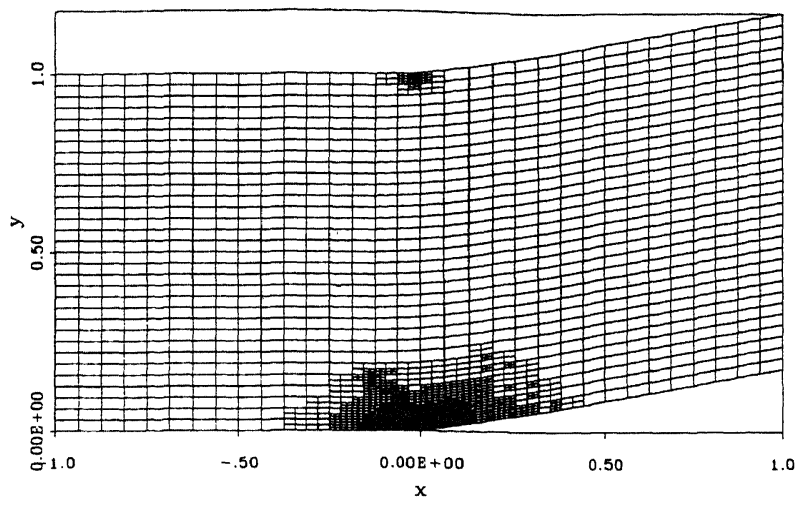

a. In full.

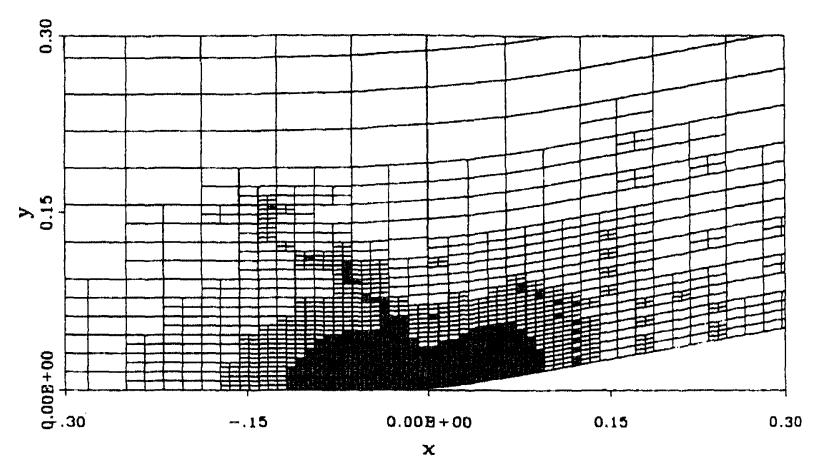

b. In detail.

Figure 19. $32 \times 32$ grid with four levels of local refinement

\section{CONCLUSIONS}

In this paper we have studied two possible causes of the zeroth-order global discretization error as encountered in the numerical approximation of subsonic Euler flow along a kinked wall: (i) non-smoothness of the grid and (ii) non-smoothness of the exact solution of the continuous Euler equations. (The approximation of the flow along a smooth wall shows, as expected, that the entropy error is not caused by the discretization of the solid wall boundary condition.)

Concerning the non-smoothness of the grid, it appears that the upwind finite volume discretization of the equations has a zeroth-order local truncation error at the kink in the grid. For supersonic flow this local truncation error can be removed by widening the stencil of the discretization in the direction in which the grid is shifted. For subsonic flow the zeroth-order local truncation error is not easily removed. However, the numerical computation of a subsonic, non-uniform, parallel flow on a kinked grid without wall suggests that the zeroth-order local truncation error does not cause the zeroth-order global discretization error.

Concerning the non-smoothness (i.e. the being singular) of the exact solution, it is well known that the incompressible potential flow along a kinked wall has a singularity at the kink. It is likely 
that a similar singularity is adopted by the Euler flow. The numerical approximation of the incompressible potential flow by means of the Euler equations with proper source term shows that a zeroth-order global discretization error arises at the same places where it is found in the approximation of the Euler flow. However, extraction of a singularity from the dependent variables of the Euler equations-a singularity which is assumed to be similar to the one in the incompressible potential flow solution - is not feasible because it does not lead to a set of equations which can be solved with the existing method.

Removing the zeroth-order global discretization error is still possible. We find the paradoxical result that by making a sequence of geometrically less accurate (but specific) discretizations of the kinked wall, a numerical solution with better error behaviour can be obtained. The less accurate discretizations of the kinked wall employ discrete smooth versions of the exact kinked wall. By making the discretization of the geometry dependent in a proper way on the mesh size $h$, an $O\left(h^{\mu}\right)$-entropy error, $0<\mu<1$, can still be obtained. Poor computational efficiency due to the rather low order of accuracy $\mu$ can be circumvented very effectively by application of local, solution-adaptive grid refinements.

\section{ACKNOWLEDGEMENTS}

The authors wish to thank P. W. Hemker and P. Wesseling for their constructive comments. This work was supported by the European Space Agency (ESA) through Avions Marcel Dassault-Bréguet Aviation (AMD-BA).

\section{REFERENCES}

1. P. W. Hemker and S. P. Spekreijse, 'Multiple grid and Osher's scheme for the efficient solution of the steady Euler equations', Appl. Numer. Math., 2, 475-493 (1986).

2. B. Koren, 'Defect correction and multigrid for an efficient and accurate computation of airfoil flows', J. Comput. Phys., 77, 183-206 (1988).

3. B. Koren, 'Euler flow solutions for transonic shock wave-boundary layer interaction', Int. j. numer. methods fuids, 9, 59-73 (1989).

4. A. Dervieux, B. van Leer, J. Périaux and A. Rizzi (eds), Proc. GAMM Workshon on the Numerical Simulation of Compressible Euler Flows, Rocquencourt, 1986; Notes on Numerical Fluid Dynamics, Vol. 26, Vieweg, Braunschweig, 1989.

5. S. Osher and F. Solomon, 'Upwind difference schemes for hyperbolic systems of conservation laws', Math. Comput., 38, 339-374 (1982).

6. H. T. M. van der Maarel, 'A solution-adaptive multigrid method for the steady Euler equations', Report NM-Rglxx, Center for Mathematics and Computer Science, Amsterdam, 1991. 